\title{
«Votre mémoire culturelle » : entre logistique numérique de la recommandation et médiation patrimoniale. Le cas de Sens Critique
}

"Your cultural memory ": from the logistics of digital recommendation to cultural heritage. The case of the Sens Critique website

Julia Bonaccorsi et Valérie Croissant

\section{(2) OpenEdition}

Journals

Édition électronique

URL : http://journals.openedition.org/edc/6467

DOI : 10.4000/edc.6467

ISSN : 2101-0366

Éditeur

Université Lille-3

\section{Édition imprimée}

Date de publication : 1 décembre 2015

Pagination : 129-148

ISBN : 978-2-917562-14-7

ISSN : $1270-6841$

Référence électronique

Julia Bonaccorsi et Valérie Croissant, « «Votre mémoire culturelle» : entre logistique numérique de la recommandation et médiation patrimoniale. Le cas de Sens Critique », Études de communication [En ligne], 45 | 2015, mis en ligne le 01 décembre 2017, consulté le 30 avril 2019. URL : http:// journals.openedition.org/edc/6467 ; DOI : 10.4000/edc.6467 
«Votre mémoire culturelle»:

entre logistique numérique de la recommandation et médiation

patrimoniale. Le cas de Sens Critique

"Your cultural memory ":

from the logistics of digital

recommendation to cultural heritage.

The case of the Sens Critique website

Julia Bonaccorsi

ELICO, Professeure en Sciences de l'information

et de la communication, Université Lyon 2

julia.bonaccorsi@univ-lyon2.fr

Valérie Croissant

ELICO, Maître de Conférences en Sciences de l'information

et de la communication, Université Lyon 2

valerie.croissant@univ-Iyon2.fr 
L'article propose une analyse des médiations des objets culturels qui s'élaborent dans des espaces médiatiques numériques relevant d'industries et d'organisations non publiques, comme les sites web participatifs de service privés. La recherche décale sensiblement le point de vue fondé sur les processus d'institution du patrimoine pour s'intéresser aux formes de médiation de la culture qui «braconnent» sur les terres des institutions médiatiques culturelles et patrimoniales. Le terrain de l'étude est constitué par le site francophone Sens Critique, qui propose aux internautes de construire et partager des parcours critiques sur les produits culturels par le croisement complexe de plusieurs systèmes d'évaluation et de recommandation.

Mots-clés : Prescription culturelle, sémiologie, médiatisation culturelle, critique culturelle, bien culturel, patrimoine.
This article analyzes the mediation of cultural objects within private sector digital spaces such as private service collaborative web sites. Rather than focussing on the processes of institution of cultural heritage, this study examines forms of cultural mediation which "poach" on the territory of heritage and cultural media institutions. A case study analysis is made of the French website Sens Critique which allows users to construct and share their own critical experience of cultural products through different assessment and recommendation systems.

Keywords: Cultural heritage, cultural mediation, digital culture, cultural criticism, semiology. 
Quelles médiations de la culture s'élaborent dans des espaces médiatiques numériques relevant d'industries et d'organisations non publiques, comme les sites web participatifs de service privés ${ }^{1}$ ? La valorisation des objets culturels dans ces sites relève à première vue d'une économie propre aux industries numériques et largement analysée aujourd'hui comme une économie de la recommandation (Beauvisage et al., 2013). Cependant, et c'est l'hypothèse principale de notre recherche, il est bien évident que cette spécificité ne peut être considérée comme imperméable à d'autres logiques de valorisation: les rapports à la culture ne sont pas renouvelés comme on endosserait un nouveau costume, et ils intègrent des formes de la relation préfigurées, suggérées par les dispositifs médiatiques et leurs architextes à des modèles préexistants. De ce fait, les statuts et valeurs des formes culturelles promettent des objets d'observation originaux. En nous intéressant à ces industries médiatiques, nous considérons qu'elles participent à modifier les représentations sociales de la culture dans le sens d'une "altération », considérant la patrimonialisation comme une transformation active des objets culturels (Jeanneret, 2014).

La visée est de nourrir la réflexion sur la dimension instituante de la patrimonialisation, c'est-à-dire qui «change le statut de l'objet en patrimoine, contribue à construire une relation entre cet objet et des personnes " (Davallon, $2006,155)$, dans des espaces de pratiques non institutionnels. L'article décale sensiblement un point de vue fondé sur les processus d'institution du patrimoine pour s'intéresser aux formes de médiation de la culture qui «braconnent » sur les terres des institutions médiatiques culturelles et patrimoniales.

Il s'agit ici de décrire et de qualifier des «écritures du patrimoine» (Tardy, 2009) en interrogeant l'interrelation d'une mémoire culturelle avec les objets culturels vus comme des produits ainsi que les glissements entre l'individuel et le commun. La problématique peut donc être formulée ainsi : en quoi peuton dire que des entreprises du numérique qui organisent une logistique de la recommandation culturelle relèvent d'un renouvellement de la médiation des objets culturels?

Le terrain de l'étude est constitué par le site francophone Sens Critique ${ }^{2}$ qui propose aux internautes de construire et partager de véritables parcours critiques sur les produits culturels par le croisement complexe de plusieurs systèmes d'évaluation et de recommandation. L'analyse de ce site articulera deux dimensions pour considérer la valeur sociale d'objets culturels élaborée dans ce que nous considérons comme un lieu de la culture (Jeanneret, 2014): la dimension socio-économique qui qualifie les logiques organisationnelles de ces industries médiatiques; la dimension sémio-discursive qui considère

1 Ce travail s'inscrit dans un projet de recherche pluridisciplinaire, "L'avis des autres. Prescriptions et jugements culturels: la force des réseaux sociaux numériques" soutenu par l'Université Lumière Lyon 2 (2014-2016).

2 http://www.senscritique.com/ 
la matérialité signifiante des formes inscrites, à la suite des travaux menés sur les écrits d'écrans comme dispositifs d'écriture et de lecture (Candel, 2008; Davallon et al., 2012).

Sur la base de l'hypothèse d'une modélisation singulière de la valorisation culturelle numérique produite par Sens Critique, présentant certains traits d'une «filiation inversée» et relevant en cela des principes de la patrimonialisation, nous explorerons successivement deux dimensions de la définition de la mémoire culturelle dans ce site. D'abord, la focale sera placée sur le dispositif médiatique, autorité énonciative et logistique d'un discours sur l'activité culturelle. Ensuite, il s'agira d'interroger les valeurs des objets culturels variant en fonction d'une ligne temporelle. Nous montrerons en quoi le site participe à des médiations des patrimoines à partir des documents qui y sont indexés et organisés en collections.

\section{1 \\ Le discours culturel du réseau social Sens Critique: le sens de la distinction}

1.1 Mes avis, mes amis: l'activité critique et le modèle du «bouche-à-oreille»

Le site web Sens Critique est créé en $2011^{3}$ et repose sur la prétention communicationnelle d'amplifier par les moyens numériques le «bouche-àoreille culturel ${ }^{4}$ ». Cette promesse est ainsi résumée sur le site: «Donnez votre avis sur la culture et découvrez les avis de vos amis. Votre collection en ligne accessible à tout moment sur Sens Critique».

Ainsi, ce réseau social suggère à chaque internaute la composition d'un profil lié à sa "consommation» culturelle passée, présente et même future. Le profil d'un membre affiche peu d'informations, l'interface valorise avant tout ses activités: il apparaît comme construisant une identité agissante plutôt que déclarative (Georges, 2008). Deux logiques sont prédominantes dans l'énonciation éditoriale du site: d'abord, celle du classement qui concerne aussi bien les produits culturels notés que les membres du réseau; ensuite, celle de la collection (figure 1).

3 Clément Apap, Kevin Kuipers et Guillaume Boutin constituent le trio fondateur, déjà à l'origine de Gamekult, revendu en 2007 pour financer Sens Critique.

4 «Nous croyons au pouvoir du bouche à oreille. Découvrez, notez, partagez avec vos amis». 


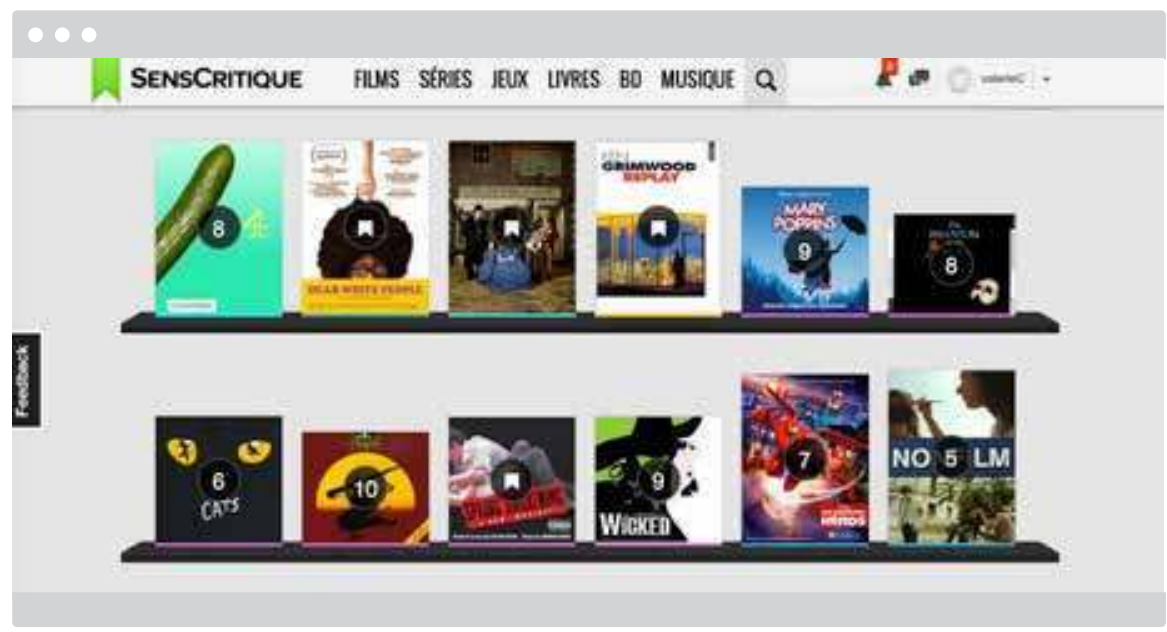

Figure 1: la collection d'un membre se présente comme une étagère sur laquelle sont disposés les produits ${ }^{5}$

La "critique» est ainsi saisie dans ces deux logiques à partir desquelles se définit un espace éditorial de mise en visibilité d'appréciation et de classement des produits culturels. Chargés de «recommandation» (vue comme une évaluation des produits), les membres du réseau sont eux-mêmes objets d'évaluation selon deux modalités: d'une part, une reconnaissance par les pairs comme "éclaireur» et par des indices de notoriété, d'autre part, l'attribution de badges validant un niveau ou par des privilèges.

Les productions discursives des membres sont ainsi fortement traversées par la gestion de leur profil et de l'identité qu'ils façonnent à travers les différentes modalités de classement et de fabrique de collections. Les membres du réseau réalisent principalement deux gestes critiques qui conditionnent l'écriture de leur profil: évaluer et réunir.

Pour des raisons économiques de vente d'espace publicitaire, mais aussi afin que chaque membre perçoive la communauté vivante, le dispositif est une incitation généralisée à être actif. On peut manifester son intérêt pour des éclaireurs, dire si l'on a apprécié une critique, noter les produits, rédiger et commenter des critiques, annoncer que l'on souhaite voir un film ou lire un ouvrage... De fait, le profil de l'utilisateur se présente comme une synthèse quantifiée des activités: il est possible de visualiser de manière graphique le nombre de critiques produites dans chacun des domaines, mais aussi la moyenne des notes du membre, la répartition de ses notes sur dix, la répartition des notes par décennies (date de sortie des produits), sa collection, ses 
listes, ses sondages... Les éclaireurs les plus actifs affichent plusieurs milliers de notes et critiques, ils sont souvent plutôt spécialisés dans un domaine ou deux (majoritairement Films, Livres et Musique), développant des pratiques de distinction. Avant de considérer plus en finesse ces listes et les collections qu'elles définissent, concentrons nous sur quelques points remarquables du dispositif médiatique que constitue le site, notamment l'agencement et la visibilité des métadonnées associées aux objets culturels.

1.2 Des produits, une vitrine: les objets culturels et leurs métadonnées

L'image constitue le signe passeur le plus important du site: malgré une interface graphique épurée, l'organisation documentaire et algorithmique est en fait d'une grande sophistication. Chaque produit est presque toujours présenté par une image (appelée "photo» dans le site): première de couverture pour les livres et bandes dessinées, affiches pour les films, pochette de l'album pour la musique. Cette iconographie carrée ou rectangulaire définit des points de repère centraux, la barre de menu principale étant constituée des rubriques «produits»: Films, Séries, Jeux, Livre, BD, Musique (voir figure 1). Notons que les catégories ne recouvrent pas de domaines culturels ni même de filières industrielles, mais désignent bien des produits culturels. L'image d'un livre équivaut à celle d'un film ou d'une série, seuls les formats varient; elle ne conduit pas à la pratique: en cliquant sur une image, on accède à la fiche documentaire de l'œuvre et aux critiques des membres mais on ne peut ni visionner un film ni lire ou même acheter directement un ouvrage.

Si les objets culturels sont les repères visuels de navigation, le principe éditorial est en revanche celui de l'évaluation, de la notation. Activées par le curseur de la souris, les images de livres, films ou jeux vidéo sont augmentées de petites bulles délivrant une information chiffrée. Signe d'une moyenne calculée, la note apparaîtra sur la «fiche» de l'œuvre culturelle associée à d'autres métriques, comme un diagramme de répartition des notes (figure 2). 


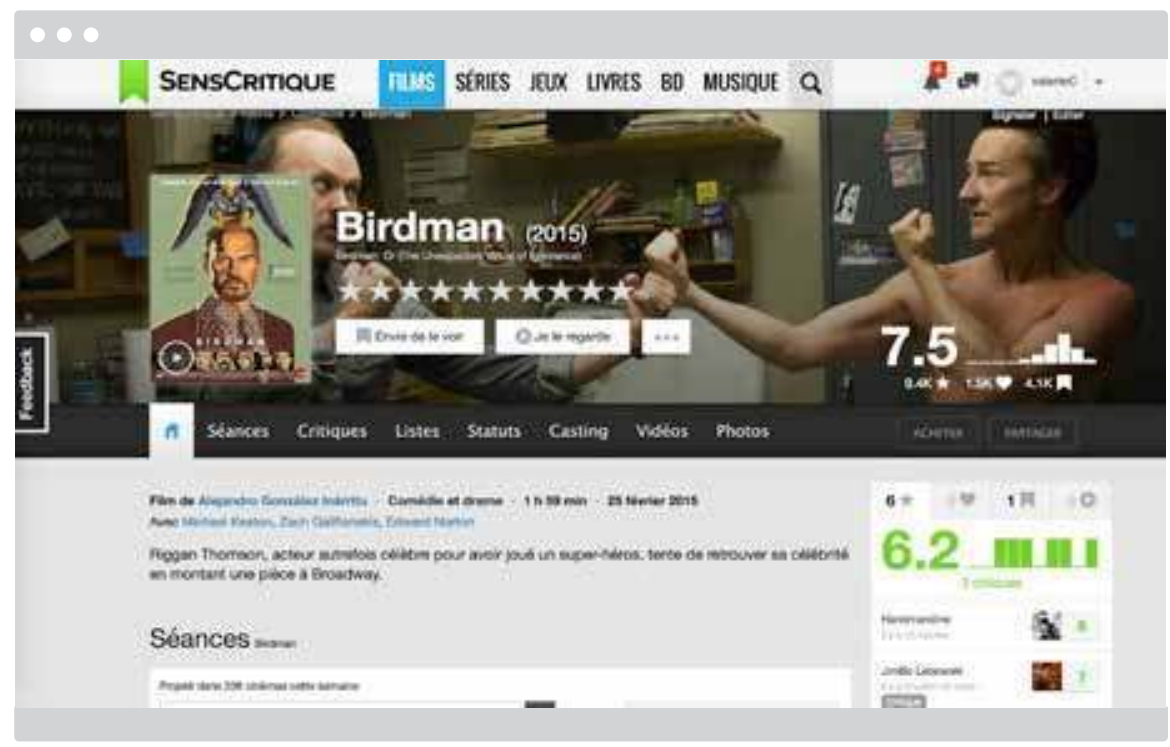

Figure 2: exemple des systèmes de notation d'un film

Les métriques en privilégiant la notation comme indice du jugement paraissent rendre plus immédiates les pratiques de construction du goût, de sélection et de distinction culturelles. Le site promet une hiérarchisation à partir de la subjectivité: il s'agit d'assurer un fonds minimum d'avis, de mobiliser, solliciter, faciliter la participation et la production de traces d'usage. Cependant, on observe que les éclaireurs les plus actifs, mais aussi les plus suivis sont ceux qui se distinguent autant par le nombre de produits connus, la durée et l'importance de leur activité, que par la longueur des textes rédigés ou leurs goûts originaux et éclectiques. Le collectif est donc un ensemble dans lequel certains individus sont en mesure de peser plus lourdement sur les métriques que d'autres ${ }^{6}$.

1.3 De la recommandation à la collection: un détournement de la valeur d'usage des produits culturels

L'analyse des métadonnées et de leur sémiotisation convergent avec les principes de recommandation économiquement valorisables caractérisés pour d'autres sites comme Allociné (Pasquier, Beaudoin, Legon, 2014). Mais, en regard d'une tendance algorithmique à la compilation et à la moyenne d'avis diversement produits, Sens Critique définit une ligne éditoriale qui tente d'échapper à cette communication-nombre produite par la statistique (Bouchard, 2008) en

6 La note unique d'un membre peu actif aura moins de poids dans le calcul que celle d'un membre actif régulièrement. Ce principe a pour objectif de limiter les pratiques de notation trop intéressées. 
mobilisant la «forme collection». Dans un article récent, Luc Boltanski et Arnaud Esquerre décrivent de manière approfondie les rapports entre des objets et la production de valeur sur eux, celle-ci constituant le dispositif de justification d'un prix. Trois formes conventionnelles (que nous pourrions appeler des médiations) permettent aux auteurs de qualifier «le type de ressource collective à laquelle les acteurs peuvent faire référence quand ils doivent s'orienter dans le monde des objets " (Boltanski, Esquerre, 2014, 27): la forme standard, la forme collection et la forme actif sont toutes trois traversées par deux axes, temporel et différentiel. Une des pistes particulièrement intéressante de ce texte concerne les décalages analysés entre la valeur d'usage et la possibilité d'accès à un statut d'œuvre; Boltanski et Esquerre interrogeant plus spécifiquement les figurations des objets du luxe à l'époque contemporaine. Le collectionneur partage la convention d'une collection idéelle car il inscrit son entreprise dans une communauté de collectionneurs par des principes de rapprochement, de régulation et des conventions.

Sans trop forcer la comparaison avec Sens critique, il est possible d'observer de quelle manière les potentialités de la forme collection sont là industrialisées par l'architexte lui-même, et remplissent les mêmes fonctions "d'économie de l'enrichissement ". La collection constitue une dimension particulière de ces modalités contrastées de la singularisation d'objets culturels: elle a comme spécificité d'être "orientée vers le passé et de se détourner de l'usage» (Boltanski, Esquerre, 2014, 30), elle masque le caractère standardisé en faisant passer des objets de l'artefact à l'œuvre parce qu'elle permet d'«envisager les choses sous le regard d'une possibilité d'accès à une sorte d'immortalité» (Boltanski, Esquerre, 2014, 38). La collection est une métaphore de la base de données dans le site Sens Critique: base de donnée qui, alimentée par les contributeurs et organisée par l'architexte du site, permet une représentation de «[notre] mémoire culturelle».

D'une part, cette logique intègre les enjeux de distinction inhérents à la capitalisation culturelle en même temps qu'une individualisation des rapports à la culture fondée sur l'actualité; d'autre part, elle énonce un lieu de conservation de sélections opérées par les contributeurs: «votre collection en ligne accessible à tout moment ». Les listes, classements, tops, produits par les membres sont compilés (au sens quasi étymologique du terme compilare: piller) par l'algorithme du site qui produit autant de synthèses. Gouvernée par le régime de l'actualité culturelle, l'énonciation éditoriale du site et ses ramifications engagent de nouvelles valeurs des collections qui déplacent les enjeux de valorisation liés à l'actualité.

Étudier les inscriptions de l'activité des internautes sur le réseau social et les diverses possibilités de classer, juger les produits culturels, mais aussi les critiques des autres membres a permis de rendre compte des modèles culturels en jeu dans les médiations éditoriales du site participatif où le palmarès occupe une place transversale et calculée. Si l'interface ne valorise pas les références 
au passé mais se centre sur l'actualité, les membres élaborent des liens avec des œuvres passées, (histoire de la bande dessinée ou du cinéma), "sur le mode d'un devenir social de la production du sens» (Davallon, 2012, 269): s'élabore ainsi une pratique de la patrimonialisation dont nous souhaitons maintenant préciser l'«allongement» des médiations éditoriales (ibid., 253). Notamment, l'axe temporel n'est pas homogène et constitue une variable importante du statut des objets culturels énoncés.

\section{2 \\ Les temps du patrimoine: du produit culturel à l'objet de collection}

Dans cette deuxième partie, nous observons plus particulièrement les temporalités énoncées par Sens Critique, et dont on peut souligner les bifurcations complexes. Boltanski et Esquerre donnent à l'axe temporel un rôle actif dans la valorisation par la forme collection. Si le cadre propositionnel du dispositif de Sens Critique repose sur l'actualité culturelle cadencée par les sorties, parutions, programmations; la notion de collection est mise en avant par le dispositif pour chaque membre comme moyen d'inscrire ses activités dans le site («votre collection»).

«Mémoriser, c'est toujours réorganiser les contenus en prenant ses distances avec leur agencement initial au profit de relations moins prévisibles» (Merzeau, 2013). La collection est un des lieux de ces ré-agencements. Nous souhaitons approfondir la manière dont le site se définit comme un lieu de pratique culturelle selon des normes qui vont donner sens et valeur aux objets culturels (Després-Lonnet, 2009). Cette chercheuse analyse ainsi la façon dont le «visiteur» recompose un ensemble d'acteurs, d'activités, de comportements, de normes pour donner sens et valeur aux objets culturels et les valider. Nous prolongeons cette analyse, mais en inversant la perspective: les objets du patrimoine ne sont pas désignés et sélectionnés a priori. Au contraire, ils sont énoncés dans les productions sémiotiques des contributeurs que l'organisation documentaire du site (sa logistique) participe à organiser en "patrimoine» notamment sur deux modes: la mémoire individuelle, les référents collectifs.

\subsection{L'effacement des signes du marchand}

Bien sûr, l'architecture documentaire du site engage une automatisation de l'archivage (ce qui va, est, a été) qui fait vivre à un même produit culturel des régimes de temporalité successifs. A priori la notion de patrimoine n'a pas sa place, écrasée par le registre de l'actualité culturelle. Cependant la critique culturelle, qu'elle soit professionnelle ou non, dans les institutions ou les marges, affirme sa légitimité dans le fait que son rôle n'est pas réductible à la production de jugements sur l'actualité culturelle, elle démontre pour ce faire sa capacité 
à porter un regard rétrospectif en manifestant une connaissance élargie ne se limitant pas au catalogue des sorties. Cette pratique de mise à distance du temps de l'actualité (dicté par les industriels de la culture) se retrouve dans Sens Critique tant au niveau du dispositif que des pratiques des membres.

La patrimonialisation est une activité qui consiste à distinguer des objets du passé ou du présent en vue de les conserver et les transmettre:

× Le patrimoine s'institue donc au présent, dans l'événement de la réception, dans la constitution d'un regard sur un objet. En ce sens, il n'est pas dissociable des communautés qui désignent un objet comme objet patrimonial, qu'il s'agisse du regard autorisé des représentants de la culture légitime [...] ou de celui des communautés d'amateurs qui ont émergé sur le web (Saemmer, Dufrêne, 2014).

Le dispositif de Sens Critique, en masquant les aspects marchands de la consommation des produits culturels, institue les participants en acteurs de la patrimonialisation, sans lien avec les instances classiques qui assurent d'ordinaire cette fonction (musées, critiques, experts, marché). Sont exclues des informations relatives à la vie sociale du produit: le site attribue aux discours des membres une place centrale, sans comparaison ni contradiction avec d'autres instances d'évaluation culturelle. Le système offre en revanche la possibilité de comparer une collection ${ }^{7}$ entre les membres du réseau. La seule dissonance permise est donc celle qui se situe à l'intérieur de l'espace du site ; elle a pour objectif de susciter des échanges entre les membres et de positionner l'expérience subjective comme axe essentiel de la production discursive. Cette relative imperméabilité du site aux autres instances critiques ou évaluatrices tend à protéger les membres d'éventuelles influences extérieures et à leur octroyer la totalité de l'espace critique. Le dispositif institue ainsi le collectif des membres comme seul juge dans son espace et lui offre des outils de construction et de mise en visibilité de ce jugement.

2.2 La liste comme écriture de la réflexivité: vers une mémoire culturelle

La liste constitue à ce titre une matrice qui peut prendre des formes variées (le répertoire, la sélection, le mémo).

7 Il faut un minimum de 200 notes pour pouvoir comparer sa collection avec un autre membre. 


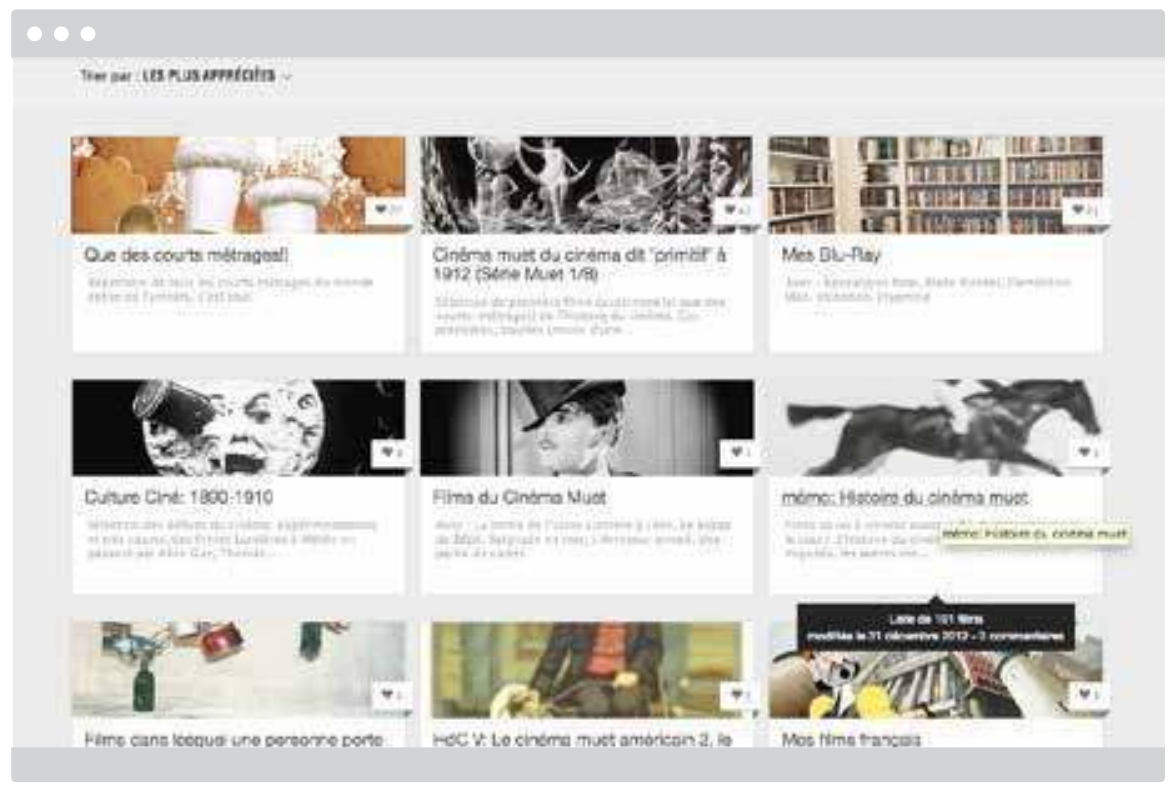

Figure 3: micro-collections en mosaïque

Si certains auteurs relèvent que la liste se caractériserait par un effacement de l'énonciateur (Sève, 2010) nous rejoignons plutôt l'avis d'Alain Rabatel qui signale l'action d'un énonciateur par le travail de choix et d'agencement "référables à une intention, ou, sinon à une intention, du moins à une signification que le lecteur attribue à l'auteur de la liste»(Rabatel, 2011, 32). Dans le cas de Sens Critique, la liste est une forme qui permet aux éclaireurs de se distinguer, par l'originalité du principe d'agencement et donc d'existence de la liste. Elle constitue un projet documentaire: «Un objet documentaire n'existe donc qu'à partir du moment où il signifie pour quelqu'un. Pointer l'action institutionnalisante du récepteur dans la définition de l'objet documentaire impose en même temps de l'appréhender à partir de son actualisation en tant qu'objet signifiant» (Courbières, 2012).

Certains modèles sont classiques comme les listes-classement de l'année: "les meilleurs», "les tops» et sont créés autant par le dispositif que par les membres; d'autres listes en revanche reflètent la personnalité du membre par l'affirmation d'une subjectivité, mais témoignent aussi de l'étendue de sa connaissance et de sa maîtrise d'un genre, d'un auteur ou d'une période: «Choix de littérature polaire», "Mon top des meilleurs films de 1942" ou encore "Je suis le seul a avoir posté une critique pour ce film». La liste constitue bien ici une collection individuelle qui explicite la place de l'énonciateur tout en la soumettant à la communauté. Les membres se manifestent en commentant ou en «aimant» la liste, participant ainsi à la notoriété de l'éclaireur. La liste exprime un point de vue, c'est une mini-collection qui va puiser dans l'immensité d'un patrimoine culturel élargi, pour en permettre une entrée facilitée. 
Le dispositif repose sur le présent de l'actualité et le futur proche par l'annonce des sorties, sur lesquelles réagissent les membres soit en notant/ critiquant soit en manifestant simplement un statut "envie de le voir» par exemple pour un film. Ces temporalités variables sont aussi la conséquence des déplacements produits par les membres du réseau social: enjoints de définir leur «mémoire culturelle», ils font alors retour sur leur socialisation culturelle. Le site devient le lieu de conservation de l'activité des contributeurs à propos de l'actualité culturelle, mais également un lieu de remémoration. Créer des synthèses, des best of: le principe de la liste participe de ces écritures réflexives d'une mémoire culturelle. Un autre exemple de cette énonciation réflexive concerne l'utilisation des documents iconographiques qui peuvent devenir, au-delà de l'illustration ou de la métonymie, des objets de collections.

Dans la figure 4, les affiches du film La Nuit du chasseur sont réunies dans un carrousel de présentation qui affiche chaque image au premier plan, la constituant en document unique.

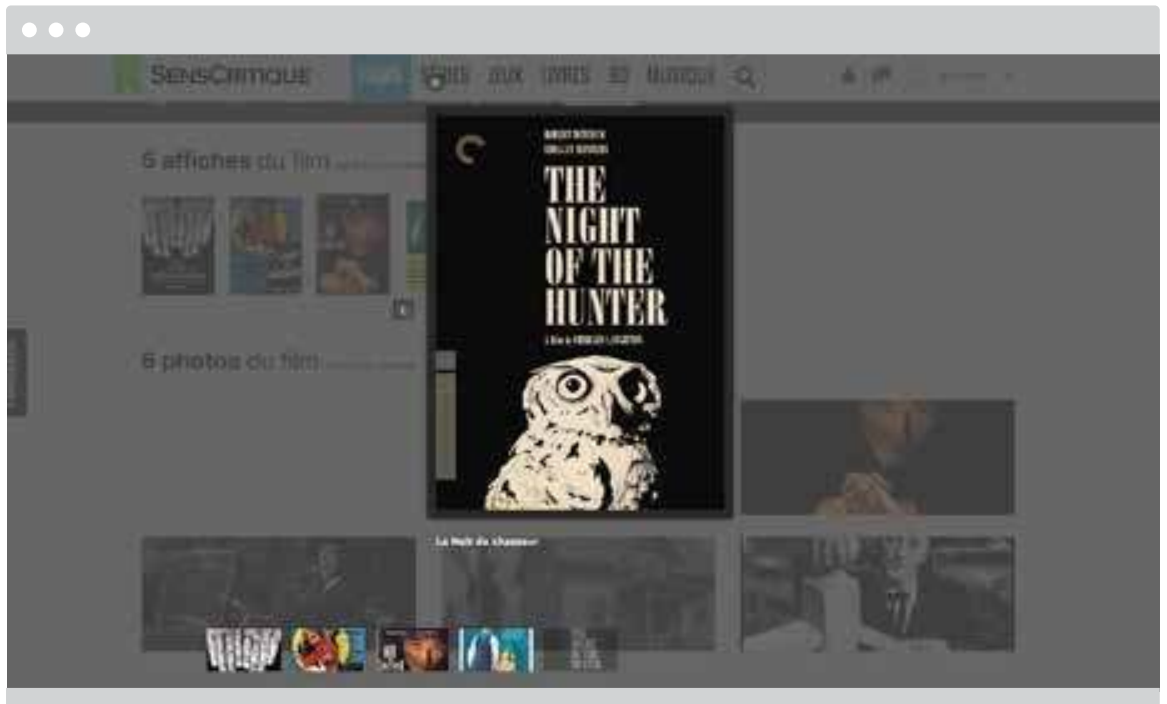

Figure 4: carrousel présentant les différentes affiches de La Nuit du chasseur, tel un corpus

La présence de plusieurs affiches du film ainsi que leur juxtaposition peuvent signifier l'importance du film, mais sans que ce discours soit assumé par une instance savante. Les internautes ont collecté ces images, le dispositif les replace dans une série, l'internaute porte la responsabilité d'attribuer un sens à cette disposition sérielle. La monstration de la diversité des ressources iconographiques mobilisées par les membres concourt à attester que le dispositif ne décide en rien du statut de ces images, que leur disposition n'est pas une forme signifiante et que tout le travail de signification appartient aux membres. Il s'agit d'une négation manifeste du rôle d'énonciation du dispositif 
dans l'objectif de mettre en évidence la force du collectif dans la production de la collection. Le métadiscours est réduit à son plus simple niveau pour donner la sensation d'un cadrage le plus détendu possible, tant et si bien que parfois les catégories mobilisées ne correspondent pas comme le montre la figure 5.

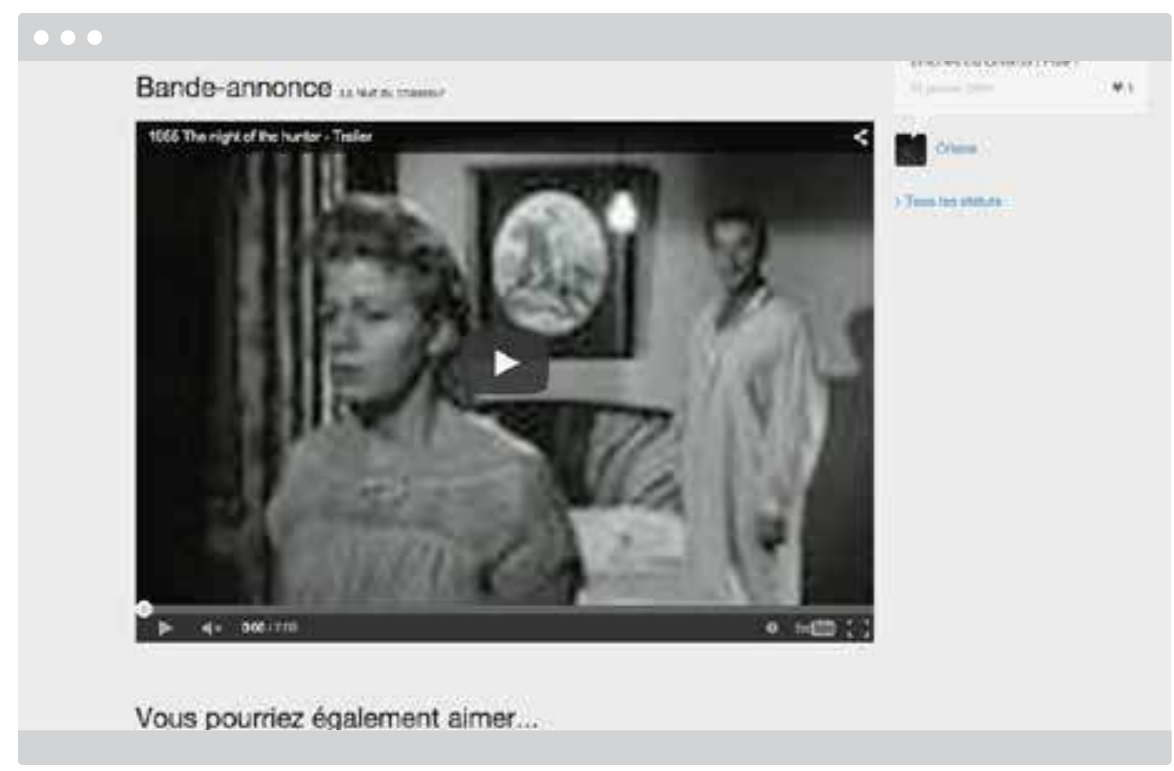

Figure 5 : l'extrait réénoncé comme une bande annonce

L'activité critique fondée sur le principe de la recommandation se transforme en autorité médiatrice qui convoque et assemble des objets culturels très variés, comme des «biens communs".

\subsection{Le Lab: la patrimonialisation par les données}

II s'agit enfin d'explorer le sens de la collection culturelle élaborée à la croisée des propriétés techno-sémiotiques du site, des formes des pratiques culturelles suggérées et observées par le site et des enjeux industriels qui conduisent le site à innover en exploitant de manière variée les bases de données constituées.

L'analyse du dispositif durant plusieurs mois ${ }^{8}$ révèle sa constante évolution. Les responsables du site ont d'ailleurs souhaité rendre traçable ce phénomène en proposant un espace d'expérimentation "Le Lab» qui propose plusieurs applications en développement. L'une d'elles se nomme «BEST OF » et «vous permet de naviguer dans les œuvres les mieux notées, année par année, ou 
décennie par décennie» concernant le cinéma. Cette application proposée en janvier 2015 compile les notes et les œuvres cinématographiques à partir des données produites par les membres en les faisant remonter sous la forme d'une chronologie des films de cinéma.

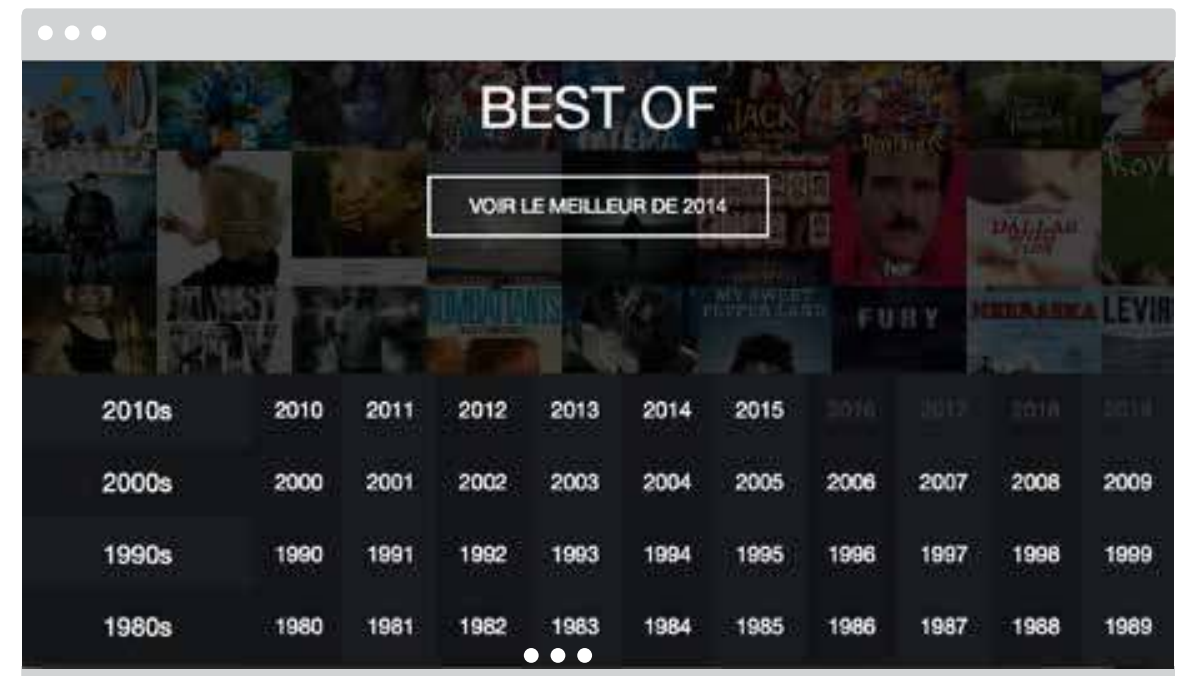

Figure 6: page d'accueil de l'application Best Of, http://lab.senscritique.com/bestof/\#/

Les images signifiant les objets filmiques (soit l'affiche, soit une image fixe du film) et leur "valeur» moyenne, apparaissent classées par ordre de taille. Le visiteur du site est donc placé face à un dispositif qui attribue une valeur à chaque film en rendant invisible le processus par lequel les notes sont produites. L'algorithme fabrique, par la collecte et une compilation visuelle des données, le niveau collectif de l'appréciation, ainsi que la temporalité longue à partir des données individuelles. II s'agit de produire une collection (sous forme d'une sélection/liste de films) à partir de données quantitatives ; la moyenne des notes attribuées valant comme point de consensus. 


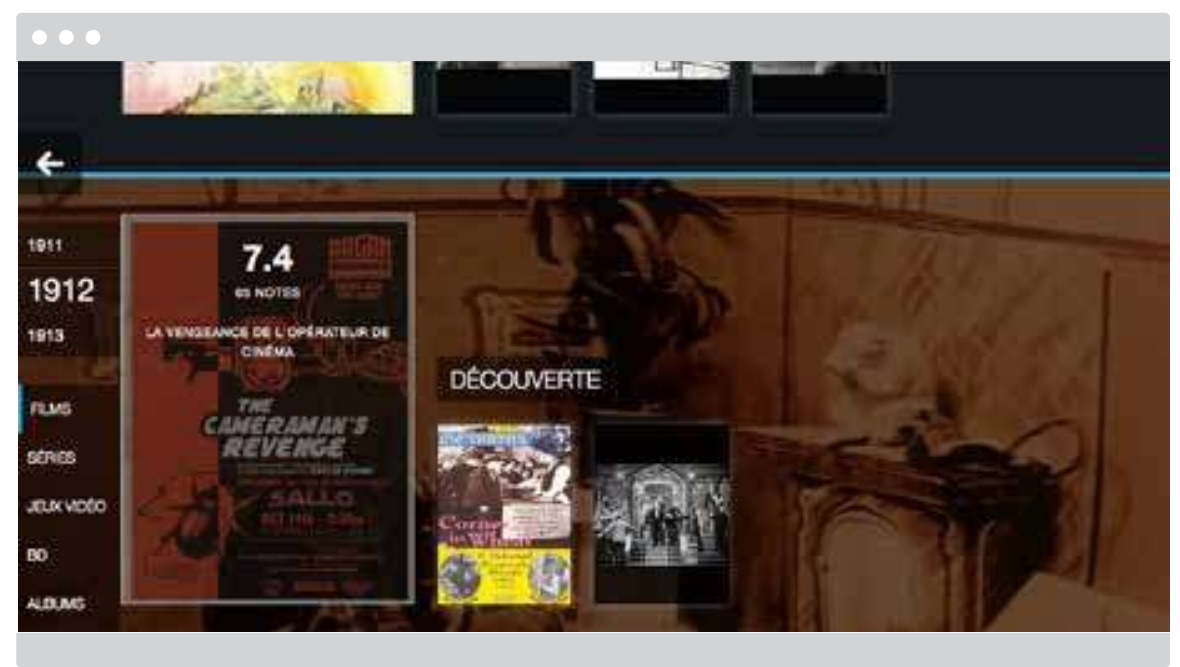

Figure 7: films référencés pour l'année 1912,

http://lab.senscritique.com/bestof/\#/year/1912/movies

Or, chaque image permet de revenir au site et aux critiques produites à son sujet: sa "fiche" (figure 7), qui comprend également, comme on le voit dans la figure 8 , la redocumentarisation du film lui-même, par une fenêtre de lecture d'un extrait long du film (plus de $12 \mathrm{mn}$ ). Ceci constitue une nouvelle entrée dans les critiques des éclaireurs, par la représentation d'une histoire du cinéma qui est loin de se limiter à l'actualité; les premiers objets culturels critiqués remontant à 1910, toutes les années et décennies étant représentées. 


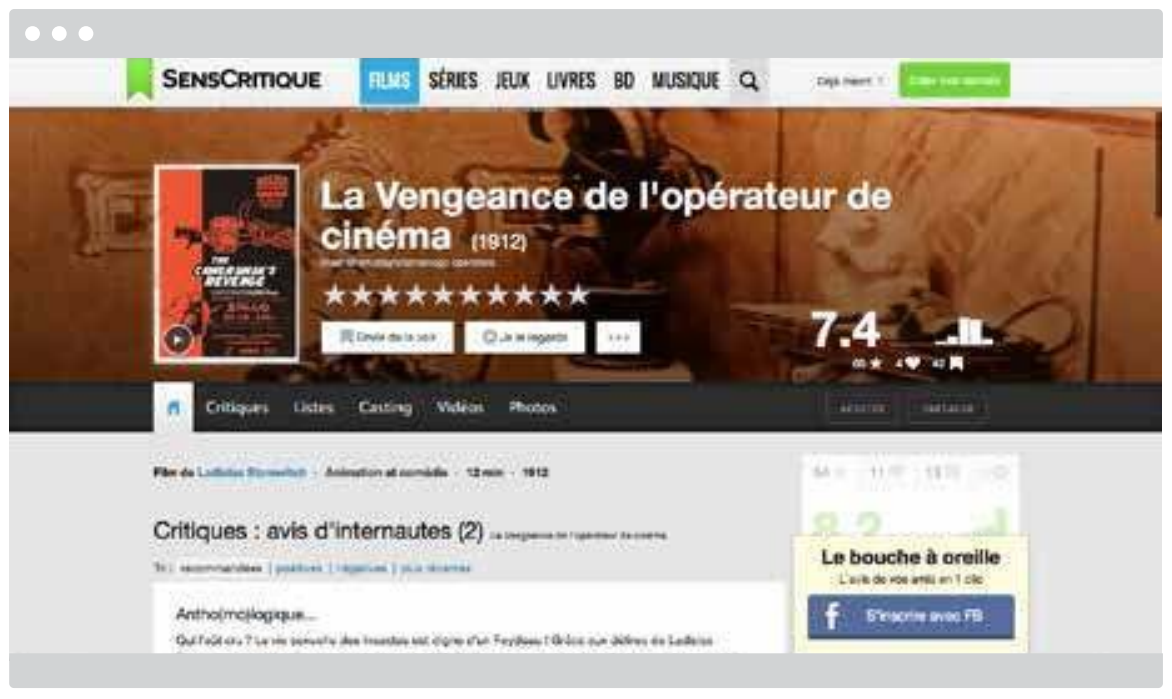

Figure 8 : fiche du film: "La vengeance de l'opérateur de cinéma»,

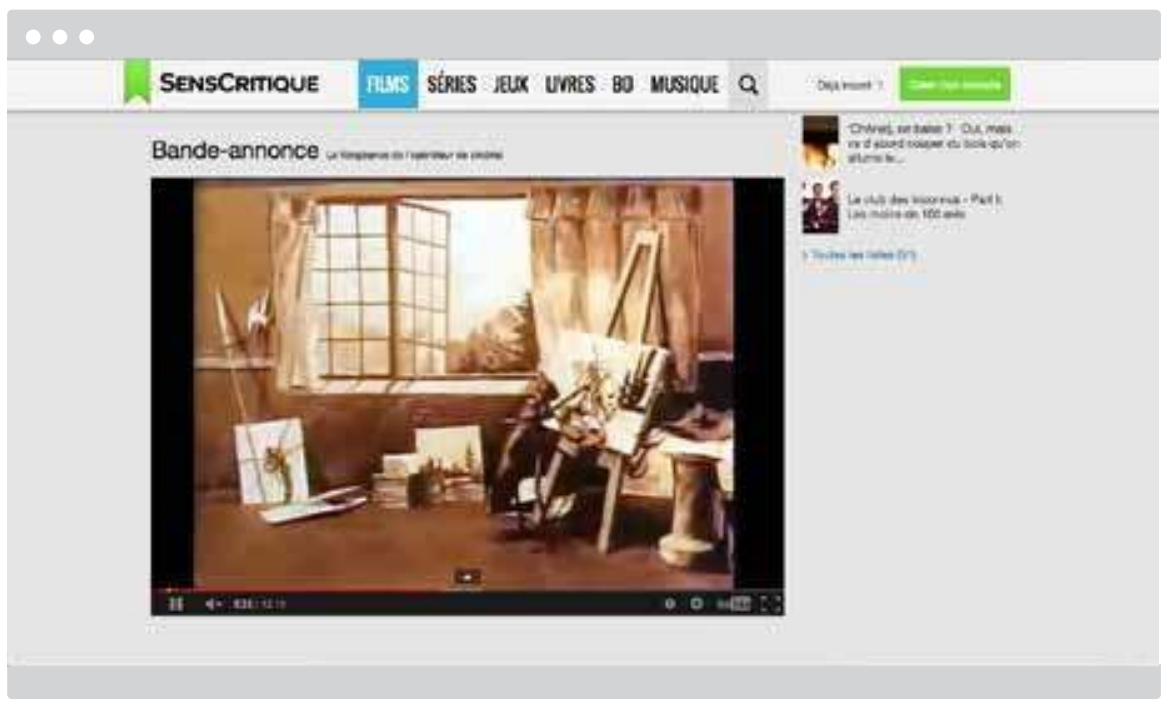

Figure 9: extrait du film à visionner 
Ainsi I'instance labellisante de ce BestOf des films est indiscernable: elle est censée référer au collectif du site, à l'ensemble des membres. Cette fabrication d'un jugement collectif à partir d'une compilation d'avis individuels repose sur un postulat fort qui consiste à voir dans l'addition et ici la moyenne des évaluations individuelles, l'évaluation du collectif. L'autre présupposé discutable est de considérer qu'une note chiffrée exprime un jugement esthétique.

Le dispositif assure ainsi par le calcul la montée en généralité: c'est lui qui prend en charge le passage des notes individuelles à une frise chronologique élargie, un temps collectif dans lequel sont gommées les individualités pour construire le temps long, le temps du commun. Dans cette économie des échanges des valeurs culturelles, nous voyons se définir une énonciation porteuse d'une nouvelle autorité, en s'appuyant sur d'autres formes d'intermédiaires que les institutions et les amateurs: ce sont les entreprises médiatiques elles-mêmes ou plutôt leurs outils. En effet le dispositif tend à rendre muette I'organisation (contrairement à un titre de presse ou un journaliste culturel) puisqu'elle délègue la «montée en généralité » des données produites par les membres aux algorithmes. Ainsi le passage de l'objet de consommation individuel à l'objet référent d'un patrimoine collectif s'effectue sur des métriques invisibles et non discutables. Nous parvenons ici semble t-il ici aux limites de I'hypothèse de la patrimonialisation, à moins de considérer les algorithmes comme l'instance instituante du collectif.

\section{3}

\section{Un processus de patrimonialisation inachevé}

La nouvelle prescription culturelle qui s'énonce dans les industries médiatiques numériques nous semble encore largement à explorer dans la diversité et les modalités contrastées avec lesquelles ces industries font usage de la «recommandation» à propos des objets culturels: industrialisation du partage de l'extrait, modèle de la critique, etc. (Beauvisage, 2013). C'est moins la figure et les axiologies du «critique», largement analysées par Étienne Candel (2008) qui ont fondé notre questionnement, qu'un intérêt porté spécifiquement sur les «objets de culture» qui sont mis en discours, à la fois dans les contributions des sujets inscrits sur la plate-forme, comme par la logistique documentaire de représentation de l'information ainsi que dans l'énonciation éditoriale, formelle et visuelle du site dans son ensemble. En étudiant un réseau social qui permet de «découvrir, noter et classer des produits culturel» nous nous sommes intéressées à la mise en discours de la culture dans des espaces revendiquant un caractère non institutionnel.

Si I'on peut sans aucun doute discerner des enjeux en lien avec des formes de patrimonialisation dans le site Sens Critique, il faut interroger les ressorts sur lesquels le processus opère. Les valeurs dévolues aux objets culturels sélectionnés ne relèvent pas seulement d'un ici et maintenant mais de la constitution d'une banque structurée de mémoire culturelle: en raison de la 
saisie formelle et numérique par le dispositif et ses algorithmes (la base de donnée) comme en raison de la description par les contributeurs qui fait l'objet d'une redocumentarisation par le média lui-même. Ces usages triviaux de la collection qui engagent un projet documentaire, participent à l'inscription de formes culturelles comme des biens communs et non plus des produits. Le média Sens Critique se positionne comme un intermédiaire culturel dont l'autorité s'expose à travers le classement élémentaire de la chronologie dans le cas du Best of. Mais le calcul peut-il prendre en charge la construction des référents culturels? C'est déjà en partie la règle dans le domaine des industries culturelles. Le site assume ainsi une position paradoxale: d'un côté il met en avant les individualités productrices des contenus sur lesquels vont s'appuyer les processus d'autorité et de légitimité. D’un autre côté la couche robotisée du site qui compile, calcule, hiérarchise les œuvres (l'étage collectif du site) est totalement invisible mais produit des signes qui affirment en permanence l'appartenance des membres à un collectif.

Les opérations de patrimonialisation observées sont ainsi élaborées à la croisée de ces logiques: elles ne sont ni le propre d'un amateurisme éclairé, ni la conséquence de la seule capacité de l'économie numérique à industrialiser les figures d'une autorité critique et légitimatrice, comme à renouveler le statut et des valeurs sociales des formes culturelles. La redocumentarisation d'un objet culturel passe par des médiations plurielles qui l'instituent selon différent degrés comme un bien commun et relèvent d'une énonciation réflexive sur la prescription culturelle. 
Bibliographie

Beauvisage T. et al. (2013). «Notes et avis des consommateurs sur le web». In Réseaux, 1, n¹77, p.131-161.

BoltanskiL., EsquerreA. (2014). "La 'collection', une forme neuve du capitalisme la mise en valeur économique du passé et ses effets". In Les Temps Modernes, $3, n^{\circ} 679$, p. 5-72.

Bouchard J. (2008). "La communication nombre. Présentation». In MEI, n²8, p.1-4.

CandelE. (2008). "L'CFuvre saisie par le réseau ». In Communication \& Langages, $n^{\circ} 155$, p. 99-114.

Casemajor-Loustau N. (2011). "La contribution triviale des amateurs sur le Web: quelle efficacité documentaire?". In Études de communication, $\mathrm{n}^{\circ} 36$, http:// edc.revues.org/index 2532. html, consulté le 13 avril 2012.

Courbières C. (2012). «Virtualité, représentation, signification: approche de la complexité documentaire». In Études de communication, $\mathrm{n}^{\circ} 39$, décembre, p.103-116.

Davallon J. (2006). Le Don du patrimoine, Cachan, Hermès Lavoisier.
Davallon J. (dir.), (2012).

L'économie des écritures sur le web, Cachan, Hermès Lavoisier.

Després-Lonnet M. (2009). "L'écriture numérique du patrimoine, de l'inventaire à l'exposition: Les parcours de la base Joconde». In Culture \& Musées, $n^{\circ} 14$, p.19-38.

DufrêneB., Gellereau M. (2004). "La médiation culturelle". In Hermès, La Revue, 1, n³8, p.199-206.

Heinich N. (2009). La fabrique du patrimoine: de la cathédrale à la petite cuillère, Éditions de la Maison des Sciences de l'Homme, coll. «Ethnologie de la France».

JeanneretY. (2014). Critique de la trivialité, Paris, Editions Non Standard.

Merzeau L. (2013). «Éditorialisation collaborative d'un événement ». In Communication \& Organisation, 1, $n^{\circ} 43$, www.cairn. info/revue-communication-etorganisation-2013-1-page-105.htm.

Pasquier D., Beaudouin V., Legon T. (2014). "Moi je lui donne 5/5». Les paradoxes de la critique amateur en ligne, Paris, Presses de I'Ecole des Mines.

Rabatel A. (2001). "Listes et effets-listes ". In Poétique 3, n¹67, p. 259-272. 
Saemmer A., Dufrêne B. (2014).

"Patrimoines éphémères». In Hybrid,

01, http://www.hybrid.univ-

paris8.fr/lodel/index.php?id=174,

consulté le 22 décembre 2014.

Tardy C. (dir.), (2009).

"L'écriture du patrimoine».

In Culture \& Musées, $n^{\circ} 14$. 